\section{Trajetórias afetivo-amorosas e perfil reprodutivo de mulheres adolescentes residentes no Município de São Paulo}

\section{Love life and reproductive profile of female adolescents residents in the City of São Paulo, Brazil}

Ana Luiza Vilela Borges 1 Néia Schor 2

1 Departamento de Enfermagem em Saúde Coletiva. Escola de Enfermagem da Universidade de São Paulo. Rua Dr. Enéas de Carvalho Aguiar, 419. Cerqueira César. São Paulo, SP, Brasil. CEP: 05. 403-000 E-mail: alvilela@usp.br 2 Departamento de Saúde Materno-Infantil. Faculdade de Saúde Pública da Universidade de São Paulo, SP, Brasil.

\begin{abstract}
Objectives: to describe the love life and reproductive profile of female adolescents between 15 and 19 years old.

Methods: a cross-sectional study comprising 222 adolescents registered in a family health clinic in the East Zone of the city of São Paulo. Home surveys were performed between June and December of 2002.

Results: among $45.5 \%$ adolescents who had already began their sexual life, the mean age of the first sexual intercourse was 15.3 years old. A considerable number (17.1\%) had already experienced p regnancy and three cases of abortion were reported. Nevertheless, at 19 years old, $2.9 \%, 14.3 \%$ and $34.3 \%$ of the adolescents had not kissed, had a boy friend or initiated a sexual life, respectively demonstrating that sexual habits in adolescence are diverse and heterogeneous.

Conclusions: adolescents reflected different reproductive profiles comprising adolescents who had never been involved in a love relationship or even sexual relationship and adolescents who had already experienced pregnancy or opted to abort. These aspects disclose the need to the design of public policies aimed at the reproductive and sexual health of Brazilian female adolescents.
\end{abstract}

Key words Pregnancy in adolescence, Teen health, Reproductive medicine

\section{Resumo}

Objetivos: descrever a trajetória afetivo-amoros a e o perfil reprodutivo de adolescentes do sexo feminino entre os 15 e 19 anos de idade.

Métodos: estudo transversal cuja população foi constituida de 222 adolescentes inscritas em uma unidade de saúde da família da zona leste do Município de São Paulo. Foram realizadas entrevistas domiciliares entre junho e dezembro de 2002.

Resultados: entre as 45,5\% adolescentes que já haviam iniciado a vida sexual, a idade média na primeira relação sexual foi 15,3 anos. Uma considerável parte $(17,1 \%)$ já havia engravidado alguma vez, tendo sido relatados três casos de aborto. Entretanto, aos 19 anos de idade, 2,9\%, 14,3\% e 34,3\% das adolescentes ainda não haviam beijado, namorado ou iniciado a vida sexual, respectivamente, mostrando que as práticas sexuais na adolescência são diversas $e$ heterogêneas.

Conclusões: as adolescentes mostraram um perfil reprodutivo diversificado, contemplando desde aquelas que nunca estiveram envolvidas em um contexto de namoro ou sequer de relacionamento sexual até aquelas que já estiveram grávidas ou optaram por um aborto, trazendo à tona a necessidade de considerar esses aspectos na elaboração de políticas públicas voltadas para a saúde reprodutiva e sexual das adolescentes brasileiras.

Palavras-chave Gravidez na adolescência, Saúde na adolescência, Medicina reprodutiva 


\section{Introdução}

Nos estudos concernentes à vida reprodutiva de mulheres jovens, a fecundidade, a gravidez e o uso de métodos contraceptivos têm ocupado lugar de destaque. É amplamente discutida a questão da intensa e rápida redução das taxas de fecundidade na população feminina brasileira como um todo, assim como o fenômeno que está na contramão dessa tendência, ou seja, o incremento na faixa etária adolescente, principalmente entre as meninas menos escolarizadas, negras e mais pobres, de regiões urbanas, fazendo com que haja um aumento na contribuição relativa das mais jovens para a fecundidade geral. 1,2

Os estudos têm apontado também que o conhecimento das jovens brasileiras acerca dos métodos anticoncepcionais pode ser considerado praticamente universal.3,4 No entanto, o seu uso, apesar de ser relativamente alto e vir aumentando, não se mostrou equivalente ao conhecimento observado. ${ }^{1}$ Isto faz com que as jovens estejam inseridas em contextos de vulnerabilidade às Doenças Sexualmente Transmissíveis (DST), AIDS e gestações não planejadas, mostrando que há, ainda, uma lacuna a ser totalmente compreendida entre o acesso à informação e a promoção de uma mudança de comportamento que comporte menos riscos à saúde. Outro fato a ser destacado é o incremento do número absoluto e relativo de gestações entre as adolescentes no país. Dados de 1994 mostram que os nascidos vivos de mulheres com menos de 20 anos de idade compreenderam $20,8 \%$ do total, enquanto que em 2000 essa proporção aumentou para 23,4\%,5 evidenciando a magnitude desse fenômeno na vida das mulheres adolescentes brasileiras.

Entretanto, alguns outros aspectos que podem ter relação com a saúde reprodutiva vêm sendo pouco abordados e explorados. Como exemplo, existem as experiências pré-sexuais, ou seja, que antecedem o início da vida sexual, como o beijar, o "ficar" e o namorar. Considerando que as adolescentes, principalmente as mais jovens, são majoritariamente solteiras, o namoro passa a ser um episódio que merece atenção, pois além de ser a relação afetiva mais comum e esperada nesse grupo, é nesse contexto que geralmente se dá o início da vida sexual, ${ }^{3}$ possibilitando que outras decorrências de uma vida sexual ativa, como as DST, AIDS, a gravidez e o aborto, sejam relacionadas também ao namoro e não apenas às uniões (legais ou consensuais) ou a relacionamentos esporádicos.

Em tal contexto, pesquisa conduzida com homens e mulheres de 18 a 24 anos de idade em três capitais brasileiras, Salvador, Rio de Janeiro e Porto Alegre, por Aquino et al. 6 , verificou que, entre as jovens que já haviam engravidado anteriormente, à primeira gravidez ocorreu com maior freqüência em relacionamento fora de uma união conjugal, sendo que as jovens ainda coabitavam com a família de origem quando engravidaram. Isto ratifica a importância dos contextos de namoro/relacionamento afetivo na vida sexual e reprodutiva de adolescentes e jovens.

Sendo o início da vida sexual um marco na vida reprodutiva de qualquer indivíduo e que ocorre com mais freqüência na adolescência, os aspectos que o antecedem e que advêm desse evento passam a ser importantes para conhecer com mais profundidade a trajetória afetivo-amorosa e reprodutiva de jovens mulheres. Este conhecimento possibilita a elaboração de políticas de saúde reprodutiva, voltadas para o grupo adolescente, que entendam seu dinamismo e heterogeneidade.

Para tanto, o presente trabalho se propõe a descrever a trajetória afetivo-amorosa e o perfil reprodutivo de adolescentes do sexo feminino entre 15 e 19 anos de idade, residentes no Município de São Paulo.

\section{Métodos}

Trata-se de um estudo transversal desenvolvido em uma amostra de jovens entre 15 e 19 anos de idade de ambos os sexos, moradores da área adscrita de uma unidade de saúde da família da zona leste do Município de São Paulo, São Paulo, Brasil. Os sujeitos entrevistados foram selecionados por amostragem sistemática, com base em uma listagem obtida por meio do Sistema de Informação da Atenção Básica (SIAB) e ordenada por microárea e pelo número das famílias cadastradas no Programa de Saúde da Família que tinham ao menos um adolescente entre 15 e 19 anos de idade. O tamanho amostral foi calculado baseando-se na precisão desejada para se estimar a porcentagem de adolescentes de 15 a 19 anos com vida sexual ativa, contemplando os objetivos do estudo maior no qual o presente trabalho está inserido. Tal proporção foi considerada igual a $60 \%, 7$ com erro máximo em valor absoluto de $5 \%$ e com nível de confiança de $95 \%$ (IC95\%). Para se obter a estimativa final do tamanho da amostra, este valor foi ajustado usando-se um fator de correção para população finita. Ainda, considerando a possibilidade de perdas, foram acrescidos $30 \%$ para que não houvesse redução do tamanho da amostra, cujo cálculo constou, finalmente, de 397 indivíduos. 
A cada quatro famílias $(K=4)$, selecionou-se um adolescente e, no caso de famílias com mais de um adolescente na faixa etária a ser estudada, um sorteio foi realizado. Para o início da seleção das famílias, sorteou-se uma entre as quatro primeiras na listagem.

Foram, então, entrevistados 406 jovens entre 15 e 19 anos de idade em seus próprios domicílios, por meio de um formulário estruturado, pré-testado e pré-codificado, entre junho e dezembro de 2002. Esta pesquisa foi aprovada pela Comissão de Ética e Pesquisa da Faculdade de Saúde Pública da Universidade de São Paulo, tendo sido garantidos os aspectos relativos ao anonimato e privacidade dos sujeitos, além da participação voluntária no estudo.

Entre os 406 jovens entrevistados, 222 (54,7\%) eram do sexo feminino e $184(45,3 \%)$ do sexo masculino. Por conta dos objetivos do presente estudo, os dados apresentados a seguir são concernentes apenas às 222 do sexo feminino entrevistadas.

Foram estudadas as seguintes variáveis em relação às adolescentes: idade média na menarca (anos), idade média no primeiro beijo (anos), idade média no primeiro namoro (anos), idade média no início da vida sexual (anos), estado conjugal, gestação anterior, gestação atual, aborto e número de filhos vivos.

Os dados foram descritos por meio de proporções e medidas de tendência central. A análise dos fatores associados à idade foi realizada por meio do teste de associação do Qui-quadrado.

\section{Resultados}

\section{Caracterização das entrevistadas}

A maior parte $(73,9 \%)$ das adolescentes era estudante. Entre essas, 9,9\% também estavam trabalhando. A ausência de inserção escolar simultaneamente à ausência de inserção no mercado de trabalho atingiu $46(20,7 \%)$ jovens. Muitas moravam em casas próprias $(66,2 \%)$ ou de aluguel $(8,6 \%)$, porém $25,2 \%$ relataram morar em habitações ocupadas. Seus domicílios possuíam em média 5,1 cômodos e 5,2 moradores.
Uma grande parte das jovens nasceu na região metropolitana de São Paulo (85,1\%), apesar do fato de que uma considerável proporção de pais e mães $(69,5 \%$ e $68,0 \%$ respectivamente) ter nascido em outras regiões do país, principalmente no Nordeste, e migrado para a cidade de São Paulo.

Em relação ao estado conjugal, 91,4\% das jovens referiram ser solteiras, $3,2 \%$ viviam em união legal, $4,5 \%$ viviam em união consensual e $0,9 \%$ já havia sido unida e encontrava-se separada no momento da entrevista.

As adolescentes tinham 16,8 anos de idade em média $(\mathrm{dp}=0,09)$ e mediana de 17,0 anos. Entre as solteiras, 39,9\% estavam namorando no momento da entrevista.

A menarca ocorreu em média aos 12,3 anos $(\mathrm{d} p=0,14)$. O primeiro beijo foi dado, em média, aos 12,2 anos de idade, com pessoas que tinham 14,6 anos de idade em média, conforme Tabela 1. Algumas jovens (4,5\%) nunca haviam beijado até o momento em que a entrevista foi realizada (Tabela 2).

O primeiro namoro ocorreu aos 13,8 anos de idade em média com indivíduos com 16,9 anos de idade. A primeira relação sexual foi em média aos 15,3 anos, com parceiros que tinham, em média, 19,6 anos de idade (Tabela 1). Cabe ressaltar que, entre todas as entrevistadas, $84,7 \%$ já haviam namorado anteriormente e $45,5 \%$ já haviam iniciado sua vida sexual. A idade foi associada estatisticamente ao início da vida sexual $(\mathrm{p}<0,001)$, resultado mostrado na Tabela 2.

Considerando as 19 jovens alguma vez unidas, os resultados mostraram que sua primeira união ocorreu aos 16,2 anos de idade com companheiros de 22,5 anos de idade, ambos valores médios (Tabela 1).

Foram relatadas 38 gestações, das quais três garotas referiram ter realizado aborto. Sete jovens relataram que não tinham nenhum filho vivo, 25 tinham apenas um filho e três tinham dois filhos (Figura 1). Nove adolescentes referiram estar grávidas no momento da entrevista, das quais três estavam grávidas pela primeira vez. 
Valores médios, desvios-padrão, medianas, valores máximos e mínimos das idades da menarca, primeiro beijo, primeiro namoro, primeira relação sexual e primeira união, seguidos dos respectivos parceiros. São Paulo, 2002.

\begin{tabular}{|c|c|c|c|c|c|}
\hline Valores & $\begin{array}{l}\text { Menarca } \\
\mathrm{N}=222\end{array}$ & $\begin{array}{l}\text { Primeiro beijo } \\
\qquad N=212\end{array}$ & $\begin{array}{c}\text { Primeiro namoro } \\
\qquad N=188\end{array}$ & $\begin{array}{l}\text { Primeira relação } \\
\qquad N=101\end{array}$ & $\begin{array}{c}\text { Primeira união } \\
\qquad N=19\end{array}$ \\
\hline \multicolumn{6}{|l|}{ Adolescentes } \\
\hline Média & 12,33 & 12,23 & 13,82 & 15,30 & 16,16 \\
\hline Desvio-padrão & 0,09 & 0,14 & 0,12 & 0,14 & 0,33 \\
\hline Mediana & 12,00 & 12,00 & 14,00 & 15,00 & 16,00 \\
\hline Valor mínimo-máximo & $8-17$ & $7-19$ & $7-14$ & $11-18$ & $14-19$ \\
\hline \multicolumn{6}{|l|}{ Parceiros } \\
\hline Média & - & 14,61 & 16,98 & 19,58 & 22,53 \\
\hline Desvio-padrão & - & 0,23 & 0,24 & 3,47 & 1,23 \\
\hline Mediana & - & 14,00 & 17,00 & 19,00 & 21,00 \\
\hline Valor mínimo-máximo & - & $7-32$ & $9-17$ & $14-30$ & $16-37$ \\
\hline
\end{tabular}

Tabela 2

Distribuição das adolescentes por idade segundo beijo anterior, namoro anterior e início da vida sexual. São Paulo, 2002.

\begin{tabular}{|c|c|c|c|c|c|c|c|c|c|c|c|c|}
\hline \multirow{3}{*}{ Variáveis } & \multicolumn{10}{|c|}{ Idade (anos) } & & \\
\hline & \multicolumn{2}{|c|}{15} & \multicolumn{2}{|c|}{16} & \multicolumn{2}{|c|}{17} & \multicolumn{2}{|c|}{18} & \multicolumn{2}{|c|}{19} & \multicolumn{2}{|c|}{ Total } \\
\hline & $\mathrm{n}$ & $\%$ & $\mathrm{n}$ & $\%$ & $\mathrm{n}$ & $\%$ & $\mathrm{n}$ & $\%$ & $\mathrm{n}$ & $\%$ & $\mathrm{n}$ & $\%$ \\
\hline \multicolumn{13}{|c|}{ Beijo anterior $(n=222)$} \\
\hline Não & 3 & 6,5 & 4 & 7,3 & 1 & 2,1 & 1 & 2,6 & 1 & 2,9 & 10 & 4,5 \\
\hline Sim & 43 & 93,5 & 51 & 92,7 & 46 & 97,9 & 38 & 97,4 & 34 & 97,1 & 212 & 95,5 \\
\hline \multicolumn{13}{|c|}{ Namoro anterior $(n=222) *$} \\
\hline Não & 14 & 30,4 & 11 & 20,0 & 2 & 4,3 & 2 & 5,1 & 5 & 14,3 & 34 & 15,3 \\
\hline Sim & 32 & 69,6 & 44 & 80,0 & 45 & 95,7 & 37 & 94,9 & 30 & 84,7 & 188 & 84,7 \\
\hline \multicolumn{13}{|c|}{ Início da vida sexual $(n=222) *$} \\
\hline Não & 40 & 87,0 & 38 & 69,1 & 16 & 34,0 & 15 & 38,5 & 12 & 34,3 & 121 & 54,5 \\
\hline Sim & 6 & 13,0 & 17 & 30,9 & 31 & 66,0 & 24 & 61,5 & 23 & 65,7 & 101 & 45,5 \\
\hline
\end{tabular}

${ }^{*} p<0,001$ (comparações entre as idades) 
Trajetória afetiva e reprodutiva das adolescentes entrevistadas antes e após a primeira relação sexual, São Paulo, 2002.

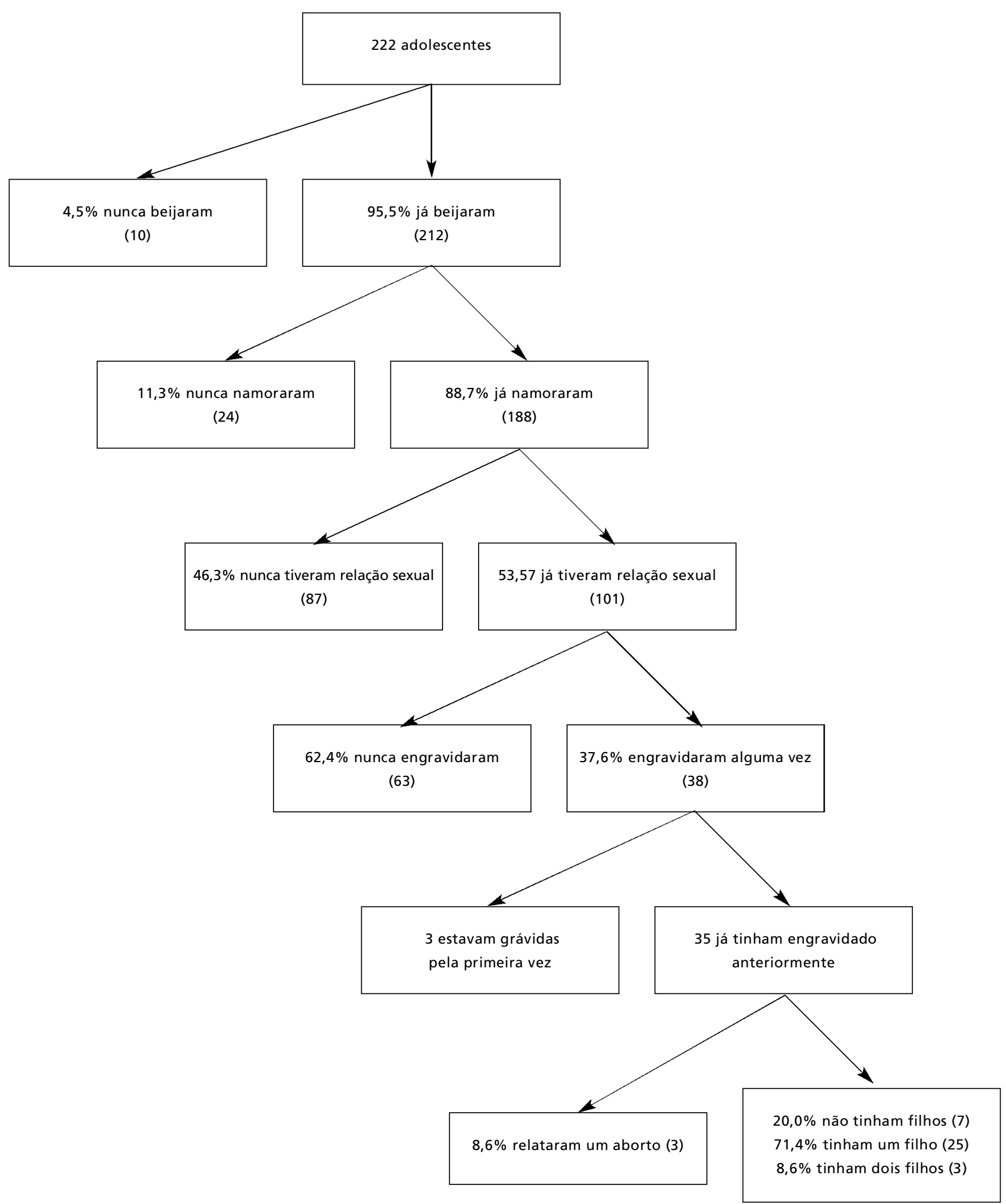




\section{Discussão}

A primeira manifestação afetivo-amorosa das garotas entrevistadas, ou seja, o primeiro beijo, ocorreu em torno da menarca, considerada o início da entrada da mulher na vida reprodutiva. Entre o primeiro beijo e o primeiro namoro passaram-se, em média, 1,6 anos e, entre o primeiro namoro e a primeira relação sexual, em média, 1,5 anos. O intervalo de tempo entre a primeira relação sexual e a primeira união foi ainda menor, 0,7 anos. Os resultados mostraram que 2,9 anos passaram-se entre a menarca e a primeira relação sexual. As jovens que revelaram ter sido alguma vez unidas levaram 2,3 anos para se unirem a partir do primeiro namoro. Esses dados são equivalentes à pesquisa conduzida por Schor $^{3}$ em uma amostra representativa de adolescentes do sexo feminino da zona Sul do Município de São Paulo em 1992, onde foi também observado que as jovens levaram 2,9 anos para terem a primeira relação sexual a partir da menarca.

No presente estudo, não está disponível a informação acerca da idade na primeira gestação, mas chama a atenção o fato de, entre as 19 jovens unidas, 17 referirem ao menos uma gestação, sugerindo que pode haver uma relação entre passar por uma gravidez e viver uma união na adolescência. Em um contexto semelhante, Schor ${ }^{3}$ relatou que as adolescentes de seu estudo que já eram mães revelaram ter engravidado pela primeira vez em torno de nove meses após a primeira relação sexual (aos 16,1 anos em média), fato condizente com a hipótese de que mulheres que engravidaram na adolescência e se uniram posteriormente, podem ter engravidado logo nas primeiras relações sexuais. Assim, salienta-se a necessidade de se prestar uma atenção especial e diferenciada à adolescente previamente ao início de sua vida sexual.

A antecipação do início da vida sexual, cuja tendência tem sido descrita na literatura, pode ser observada comparando os dados apresentados no presente estudo, cuja proporção de jovens que já haviam iniciado a vida sexual correspondeu a $45,5 \%$, dado mais próximo ao encontrado por Olinto e Galvão, 8 que foi $45,2 \%$, em mulheres da mesma faixa etária, moradoras da cidade de Pelotas, Rio Grande do Sul. No entanto, este valor foi superior aos resultados descritos por Ferraz e Ferreira ${ }^{9}$ que, ao analisarem os dados da Pesquisa Nacional de Demografia e Saúde (PNDS) de 1996, encontraram $33 \%$ das jovens brasileiras entre 15 e 19 anos de idade já iniciadas sexualmente.

As adolescentes que já haviam passado por uma gravidez totalizaram $17,1 \%$ (ou $37,6 \%$ se conside- radas apenas as que já haviam iniciado a vida sexual), percentual similar aos resultados encontrados por Ferraz e Ferreira, ${ }^{9}$ que foi $18 \%$ para população brasileira como um todo. Esses autores também observaram que há uma maior proporção de jovens grávidas ou mães entre as adolescentes com níveis mais baixos de escolaridade, justamente o perfil das adolescentes deste estudo. No Estado de São Paulo, uma pesquisa da Sociedade Civil BemEstar Familiar no Brasil (BEMFAM) 10 constatou que $17,6 \%$ das adolescentes de 15 a 19 anos já tinham estado grávidas alguma vez. Aos 15 anos de idade, essa proporção foi $4,4 \%$ e, aos 19 anos, esse percentual elevou-se para $34,8 \%$, mostrando que a maior parte das gestações na adolescência havia ocorrido entre os 15 e 19 anos de idade.

Chama a atenção o fato de que embora apenas três jovens tenham referido a realização de um aborto, sete jovens, que relataram já ter engravidado, não possuíam filhos no momento da entrevista, levando a crer que, na verdade, o número de abortos realizados pode ter sido maior. Já em 1984, Schor11 encontrou indícios de uma tendência maior de que as gestações em mulheres com menos de 20 anos de idade terminassem em aborto do que as outras faixas etárias posteriores. Para Silva,12 a população de mulheres adolescentes, solteiras, sem filhos e iniciando a vida reprodutiva nessa fase corresponde ao segmento mais exposto ao risco de abortar. Em um estudo conduzido com 620 mulheres admitidas em um hospital público por aborto incompleto, Fonseca et al. 13 verificaram que $29,7 \%$ das mulheres que relataram ter provocado aborto tinham idade inferior a 19 anos, proporção menor somente em relação à faixa etária entre 20 e 24 anos (31,9\%). Sendo o aborto permitido somente em casos especiais, sua prática, principalmente entre as mulheres mais desfavorecidas, ocorre de forma insegura e exposta a riscos à saúde, deve pois, ser objeto de investimento para o cumprimento da integralidade dos direitos reprodutivos.

Outro aspecto que merece atenção é a idade dos primeiros parceiros das jovens nos diversos eventos relacionados. Em todos esses eventos, os primeiros parceiros eram mais velhos do que as adolescentes, e a diferença entre as idades aumentava de acordo com a seriedade do evento, ou seja, no primeiro beijo, os parceiros eram apenas dois anos mais velhos e, na primeira união os parceiros eram aproximadamente seis anos mais velhos do que as adolescentes. Esse é um fenômeno a ser considerado, pois tal diferença de idade pode acarretar perdas no poder de negociação e autonomia de decisão tanto em relação ao momento de iniciar a vida sexual quanto na escolha 
do uso e tipo de métodos anticonceptivos pelas adolescentes, coerentemente com o que sugeriu Longo. 14 Nesse contexto, Aquino et al. 6 chamaram a atenção para a mais alta ocorrência de gravidez antes dos 20 anos de idade verificada quanto mais velho havia sido o parceiro de iniciação sexual das jovens de 18 a 24 anos de idade. Tal resultado também reforça o tradicional relacionamento de mulheres brasileiras com parceiros mais velhos 2 e, por conseqüência, mais experientes sexualmente e, provavelmente, mais expostos aos riscos de contrair ou ter contraído DST e AIDS.

Tanto o beijar, quanto o namorar e o "transar" são fenômenos que ocorrem pela primeira vez com mais freqüência na adolescência e pode-se observar que acontecem de forma gradual. O beijo e o namoro são eventos que ocorrem pela primeira vez nos primeiros anos da adolescência, entre 10 e 14 anos, e a primeira relação a partir dos 15 anos de idade, sendo que, neste estudo, foi observado um incremento intenso na proporção de iniciação sexual quando comparadas as garotas de $15(13,0 \%), 16$ $(30,9 \%)$ e $17(66,0 \%)$ anos de idade. Esses resultados são importantes para refletir que o aspecto idade deve ser levado em consideração pelos profissionais de saúde que trabalham com adolescentes.

A maior parte dos estudos e ações programáticas divide os adolescentes nas faixas etárias entre 10 a 14 anos e 15 a 19 anos de idade, o que, se por um lado, permite comparações entre esses grupos, por outro lado, traz limitações ao entendimento de como o processo de formação da identidade social, moral, cultural e sexual se dá. Um ano ou mesmo meses podem ser suficientes para vislumbrarem-se as modificações das decisões e atitudes de adolescentes frente às práticas sexuais, não somente pela quantidade de informações as quais o grupo tem acesso, mas, também, pela maturidade física e psicológica que ocorre continuamente no decorrer do tempo.
Mesmo assim, é importante ressaltar que tais eventos não se constituem em regra para todas as adolescentes. Aos 19 anos de idade, 2,9\%, 14,3\% e $34,3 \%$ das jovens entrevistadas não haviam ainda experienciado um beijo, um namoro ou a primeira relação, respectivamente. Isso traz a reflexão que a adolescência é, por si só, uma fase bastante heterogênea e tais diferenças devem ser consideradas ao elaborar e implementar atividades educativas que promovam a saúde. Não se deve, pois, generalizar condutas de comportamento afetivo-sexual em adolescentes apenas por conta de dados que descrevem a idade média de início da vida sexual. Lembramos que, apesar da idade média de início sexual encontrada neste estudo ter sido 15,3 anos, $54,5 \%$ das entrevistadas ainda não haviam tido nenhuma relação sexual, ratificando que, nesse grupo etário, as atitudes e práticas frente à sexualidade são diversas.

Contudo, faz-se necessário ressaltar a importância do conhecimento acerca da idade de iniciação sexual nos diversos grupos etários e sociais ao longo do tempo, no intuito de que ações educativas voltadas ao controle da fecundidade e prevenção de DST e AIDS sejam ofertadas prévia e concomitantemente ao início da vida sexual, permitindo que as jovens vivam plenamente sua vida sexual e reprodutiva mediante escolhas próprias responsáveis e sem riscos.

Concluindo, as jovens participantes do presente estudo têm um perfil reprodutivo bastante diverso, contemplando desde aquelas que nunca estiveram envolvidas em um contexto de namoro ou sequer de relacionamento sexual até aquelas que já estiveram grávidas, tiveram filhos ou optaram por um aborto, trazendo à tona a necessidade de considerar esses aspectos tanto na elaboração de políticas públicas quanto na implementação de atividades educativas e de assistência voltadas à saúde reprodutiva e sexual das adolescentes brasileiras. 


\section{Referências}

1. Yazaki LM, Morell MGG. Fecundidade é antecipada. In: SEADE. 20 anos no ano 2000: estudos sócio-demográficos sobre a juventude paulis ta. São Paulo; 1998. p. 106- 18.

2. Berquó E. O rejuvenescimento da fecundidade. Disponível em: URL: http://www.cebrap.org.br/pesqui pop_soc.htm [2004 fev 18].

3. Schor N. Adolescência e anticoncepção - conhecimento e uso. São Paulo [tese livre-docência]. São Paulo: Faculdade de Saúde Pública da Universidade de São Paulo; 1995.

4. Morell MGG, Yazaki LM. Anticoncepção e preferências reprodutivas. In: SEADE. 20 anos no ano 2000: estudos sócio-demográficos sobre a juventude paulista. São Paulo; 1998. p. 136-48.

5. Ministério da Saúde. DATASUS. Disponível em: URL: http://tabnet.datasus.gov.br/cgi/sinasc/nvmap.htm [2004 fev 25].

6. Aquino EML, Heilborn ML, Knauth D, Bozon M, Almeida MC, Araújo J, Menezes G. Adolescência e reprodução no Brasil: a heterogeneidade dos perfis sociais. Cad Saúde Pública 2003; 19 [ Supl 2]: S377-S88.

7. Ministério da Saúde. Pesquisa sobre comportamento sexual e percepções da população brasileira sobre HIV/AIDS Brasília (DF): Coordenação Nacional de DST e AIDS; 2000.

8. Olinto MTA, Galvão LW. Características reprodutivas de mulheres de 15 a 49 anos: estudos comparativos e planejamento de ações. Rev Saúde Pública 1999; 33: 64- 72.
9. Ferraz EA, Ferreira IQ. Início da atividade sexual e características da população adolescente que engravida. In: Vieira EM, Fernandes MEL, Bailey P, McKay A, organizadores. Anais do Seminário Gravidez na Adoles cência. Rio de Janeiro; 1998. p. 47-56.

10. BEMFAM (Sociedade Civil de Bem-Estar Famíliar no Brasil). Brasil-Pesquisa Nacional sobre Demografia e Saúde: 1996. R io de Janeiro; 1997.

11. Schor N. Aborto como questão de saúde pública: estudo da demanda de mulheres que recorreram ao hospital por complicações de aborto. [tese doutorado]. São Paulo: Faculdade de Saúde Pública da Universidade de São Paulo; 1984.

12. Silva RS. Recorrência ao aborto provocado. In: SEADE: 20 anos no ano 2000: estudos sócio-demográficos sobre a juventude pa ulista. São Paulo; 1998. p. 162-3.

13. Fonseca W, Misago C, Freitas P, Santos E, Fernandes L, Correia L. Características sócio-demográficas, reprodutivas e médicas de mulheres admitidas por aborto em hospital da Região Sul do Brasil. Cad Saúde Pública 1998; 14: 279-86.

14. Longo LAFB. Juventude e contracepção: um estudo dos fatores que influenciam o comportamento contra ceptivo das jovens brasileiras de 15 a 24 anos. Rev Bras Est Pop 2002; 19: 228-47.

15. Berquó E. Quando, como e com quem se casam os jovens brasileiros. In: CNPD (Comissão Nacional de População e Desenvolvimento). Jovens acontecendo na trilha das políticas públicas. Brasília (DF): 1998. v. 1, p. 93-107.

Recebido em 4 de maio de 2004

Versão final apresentada em 22 de março de 2005

Aprovado em 29 de março de 2005 